Received by the editors: December 21, 2020; Accepted: April 22, 2021

\title{
ON CERTAIN SUBCLASSES OF UNIVALENT FUNCTIONS OF COMPLEX ORDER ASSOCIATED WITH PASCAL DISTRIBUTION SERIES
}

\author{
Bilal ŞEKER and Sevtap SÜMER EKER \\ Department of Mathematics, Faculty of Science, Dicle University, Diyarbakır, TURKEY
}

ABSTRACT. In this study, by establishing a connection between normalized univalent functions in the unit disc and Pascal distribution series, we have obtained the necessary and sufficient conditions for these functions to belong to some subclasses of univalent functions of complex-order. We also determined some conditions by considering the integral operator for these functions.

\section{INTRODUCTION}

Let $\mathcal{A}$ stand for the standard class of analytic functions of the form

$$
f(z)=z+\sum_{k=2}^{\infty} a_{k} z^{k}, \quad z \in \mathbb{U}=\{z \in \mathbb{C}:|z|<1\} .
$$

Moreover, let $\mathcal{S}$ be the class of functions in $\mathcal{A}$, which are univalent in $\mathbb{U}$ (see [5]).

The necessary and sufficient condition for a function $f \in \mathcal{A}$ to be called starlike of complex order $\gamma\left(\gamma \in \mathbb{C}^{*}=\mathbb{C} \backslash\{0\}\right)$ is $\frac{f(z)}{z} \neq 0, z \in \mathbb{U}$, and

$$
\operatorname{Re}\left\{1+\frac{1}{\gamma}\left(\frac{z f^{\prime}(z)}{f(z)}-1\right)\right\}>0, \quad(z \in \mathbb{U})
$$

We denote the class of these functions with $\mathcal{S}^{*}(\gamma)$. The class $\mathcal{S}^{*}(\gamma)$ introduced by Nasr and Aouf [10].

The necessary and sufficient condition for a function $f \in \mathcal{A}$ to be called convex function of order $\gamma\left(\gamma \in \mathbb{C}^{*}\right)$, that is $f \in \mathcal{C}(\gamma)$ is $f^{\prime}(z) \neq 0$ in $\mathbb{U}$ and

$$
\operatorname{Re}\left\{1+\frac{1}{\gamma}\left(\frac{z f^{\prime \prime}(z)}{f^{\prime}(z)}\right)\right\}>0, \quad(z \in \mathbb{U}) .
$$

2020 Mathematics Subject Classification. Primary 30C45, 30C50, 30C55.

Keywords and phrases. Univalent functions, complex order, Pascal distribution, coefficient bounds, coefficient estimates.

\bilal.seker@dicle.edu.tr; sevtaps@dicle.edu.tr-Corresponding author

(D) 0000-0003-1777-8145; 0000-0002-2573-0726. 
The class $\mathcal{C}(\gamma)$ was introduced by Wiatrowski [15. It follows from 22) and (3) that for a function $f \in \mathcal{A}$ we have the equivalence

$$
f \in \mathcal{C}(\gamma) \Leftrightarrow z f^{\prime} \in \mathcal{S}^{*}(\gamma) .
$$

For a function $f \in \mathcal{A}$, we say that it is close-to-convex function of order $\gamma(\gamma \in$ $\left.\mathbb{C}^{*}\right)$, that is $f \in \mathcal{R}(\gamma)$, if and only if

$$
\operatorname{Re}\left\{1+\frac{1}{\gamma}\left(f^{\prime}(z)-1\right)\right\}>0, \quad(z \in \mathbb{U}) .
$$

The class $\mathcal{R}(\gamma)$ was studied by Halim [6] and Owa 11].

Let $\mathcal{T} \subset \mathcal{A}$ represent the functions of the form

$$
f(z)=z-\sum_{k=2}^{\infty} a_{k} z^{k}, \quad\left(a_{k} \geq 0\right) .
$$

Many important results for the class $\mathcal{T}$ have been given by Silverman [14. A lot of consequences have obtained by researchers about the functions in the class $\mathcal{T}$. Using the functions of the form $f(z)=z-\sum_{k=n+1}^{\infty} a_{k} z^{k}$, Altintaş et al. 2 defined following subclasses of $\mathcal{A}(n)$, which generalizes the results of Nasr et al. and Wiatrowski [10, 15, and obtained several results for this class. It is clear that for $n=1$, we obtain the class $\mathcal{T}$.

Definition 1. [2] Let $\mathcal{S}_{n}(\gamma, \lambda, \beta)$ denote the subclass of $\mathcal{T}$ consisting of functions $f$ which satisfy the inequality

$$
\begin{gathered}
\left|\frac{1}{\gamma}\left(\frac{z f^{\prime 2} f^{\prime \prime}(z)}{\lambda z f^{\prime}(z)+(1-\lambda) f(z)}-1\right)\right|<\beta, \\
\left(z \in \mathbb{U}, \gamma \in \mathbb{C}^{*}, 0<\beta \leq 1,0 \leq \lambda \leq 1\right) .
\end{gathered}
$$

Also let $\mathcal{R}_{n}(\gamma, \lambda, \beta)$ denote the subclass of $\mathcal{T}$ consisting of functions $f$ which satisfy the inequality

$$
\begin{gathered}
\left|\frac{1}{\gamma}\left(f^{\prime}(z)+\lambda z f^{\prime \prime}(z)-1\right)\right|<\beta, \\
\left(z \in \mathbb{U}, \gamma \in \mathbb{C}^{*}, 0<\beta \leq 1,0 \leq \lambda \leq 1\right) .
\end{gathered}
$$

We note that

$$
\mathcal{S}_{n}(\gamma, 0,1) \subset \mathcal{S}_{n}^{*}(\gamma) \quad \text { and } \quad \mathcal{R}_{n}(\gamma, 0,1) \subset \mathcal{R}_{n}(\gamma)
$$

Recently, it has been established a power series that its coefficients were probabilities of the elementary distributions such as Poisson, Pascal, Binomial, etc. Many researchers have obtained several results about some subclasses of univalent functions using these series. (see, for example $[1,3,7,8,8,9,12,13$, ) 
A variable $\mathrm{x}$ is said to have the Pascal distribution if it takes on the values $0,1,2,3, \ldots$ with the probabilities $(1-q)^{r}, \frac{q r(1-q)^{r}}{1 !}, \frac{q^{2} r(r+1)(1-q)^{r}}{2 !}$, $\frac{q^{3} r(r+1)(r+2)(1-q)^{r}}{3 !}, \ldots$, respectively, where $q$ and $r$ are parameters. Hence

$$
P(X=k)=\left(\begin{array}{c}
k+r-1 \\
r-1
\end{array}\right) q^{k}(1-q)^{r}, \quad k \in\{0,1,2, \ldots\} .
$$

Recently, El-Deeb et al. 4] introduced the following power series whose coefficients are probabilities of the Pascal distribution and stated some sufficient conditions for the Pascal distribution series and other related series to be in some subclasses of analytic functions.

$$
\begin{gathered}
\mathbf{K}_{q}^{r}(z):=z+\sum_{k=2}^{\infty}\left(\begin{array}{c}
k+r-2 \\
r-1
\end{array}\right) q^{k-1}(1-q)^{r} z^{k} \\
(z \in \mathbb{U} ; r \geq 1 ; 0 \leq q \leq 1) .
\end{gathered}
$$

Now let us introduce the following new power series whose coefficients are probabilities of the Pascal distribution.

$$
\begin{gathered}
\boldsymbol{\Phi}_{q}^{r}(z):=2 z-\mathbf{K}_{q}^{r}(z)=z-\sum_{k=2}^{\infty}\left(\begin{array}{c}
k+r-2 \\
r-1
\end{array}\right) q^{k-1}(1-q)^{r} z^{k} \\
(z \in \mathbb{U} ; r \geq 1 ; 0 \leq q \leq 1) .
\end{gathered}
$$

It is clear that $\boldsymbol{\Phi}_{q}^{r}(z)$ is in the class $\mathcal{T}$. Note that, by using ratio test we deduce that the radius of convergence of the power series $\mathbf{K}_{q}^{r}(z)$ and $\boldsymbol{\Phi}_{q}^{r}(z)$ are infinity.

We will need the following Lemmas from Altıntaş et al. 2 to prove our main results.

Lemma 2. [2] Let the function $f \in \mathcal{A}(n)$, then $f$ is in the class $\mathcal{S}_{n}(\gamma, \lambda, \beta)$ if and only if

$$
\sum_{k=n+1}^{\infty}[\lambda(k-1)+1](k+\beta|\gamma|-1) a_{k} \leq \beta|\gamma|
$$

Lemma 3. [2] Let the function $f \in \mathcal{A}(n)$, then $f$ is in the class $\mathcal{R}_{n}(\gamma, \lambda, \beta)$ if and only if

$$
\sum_{k=n+1}^{\infty} k[\lambda(k-1)+1] a_{k} \leq \beta|\gamma| .
$$

Throughout this paper, we suppose that $n=1$ for the functions in the classes $\mathcal{S}_{n}(\gamma, \lambda, \beta)$ and $\mathcal{R}_{n}(\gamma, \lambda, \beta)$ and we will write $\mathcal{S}_{1}(\gamma, \lambda, \beta)=\mathcal{S}(\gamma, \lambda, \beta)$ and $\mathcal{R}_{1}(\gamma, \lambda, \beta)=$ $\mathcal{R}(\gamma, \lambda, \beta)$ for briefly.

In the present paper, we established necessary and sufficient conditions for the functions that coefficients consist of Pascal distribution series to be in $\mathcal{S}(\gamma, \lambda, \beta)$ and $\mathcal{R}(\gamma, \lambda, \beta)$. Also, we studied similar properties for integral transforms related to these series. 


\section{Main Results}

Theorem 4. $\boldsymbol{\Phi}_{q}^{r}(z)$ given by $(6)$ is in the class $\mathcal{S}(\gamma, \lambda, \beta)$ if and only if

$$
\frac{q^{2} r(r+1) \lambda}{(1-q)^{2}}+\frac{q r(\lambda \beta|\gamma|+\lambda+1)}{1-q} \leq \beta|\gamma|(1-q)^{r} .
$$

Proof. To prove that $\mathbf{\Phi}_{q}^{r} \in \mathcal{S}(\gamma, \lambda, \beta)$, according to Lemma 2 , it is sufficient to show that

$$
\sum_{k=2}^{\infty}[\lambda(k-1)+1](k+\beta|\gamma|-1)\left(\begin{array}{c}
k+r-2 \\
r-1
\end{array}\right) q^{k-1}(1-q)^{r} \leq \beta|\gamma| .
$$

We will use the following very known relation

$$
\sum_{k=0}^{\infty}\left(\begin{array}{c}
k+r-1 \\
r-1
\end{array}\right) q^{k}=\frac{1}{(1-q)^{r}}, 0 \leq q \leq 1 .
$$

and the corresponding ones obtained by replacing the value of $r$ with $r-1, r+1$ and $r+2$ in our proofs.

By making calculations on the left hand side of the inequality 10 we obtain,

$$
\begin{aligned}
& \sum_{k=2}^{\infty}[\lambda(k-1)+1](k+\beta|\gamma|-1)\left(\begin{array}{c}
k+r-2 \\
r-1
\end{array}\right) q^{k-1}(1-q)^{r} \\
& =(1-q)^{r}\left[\sum_{k=2}^{\infty}\left(\begin{array}{c}
k+r-2 \\
r-1
\end{array}\right) q^{k-1} \lambda(k-1)(k-2)+\sum_{k=2}^{\infty}\left(\begin{array}{c}
k+r-2 \\
r-1
\end{array}\right) q^{k-1} \beta|\gamma|\right. \\
& \left.+\sum_{k=2}^{\infty}\left(\begin{array}{c}
k+r-2 \\
r-1
\end{array}\right) q^{k-1}(k-1)(\lambda \beta|\gamma|+\lambda+1)\right] \\
& =(1-q)^{r}\left[q^{2} \sum_{k=3}^{\infty}\left(\begin{array}{c}
k+r-2 \\
r+1
\end{array}\right) q^{k-3} \lambda r(r+1)+\sum_{k=2}^{\infty}\left(\begin{array}{c}
k+r-2 \\
r-1
\end{array}\right) q^{k-1} \beta|\gamma|\right. \\
& \left.+q \sum_{k=2}^{\infty}\left(\begin{array}{c}
k+r-2 \\
r
\end{array}\right) q^{k-2} r(\lambda \beta|\gamma|+\lambda+1)\right] \\
& =(1-q)^{r}\left[q^{2} \sum_{k=0}^{\infty}\left(\begin{array}{c}
k+r+1 \\
r+1
\end{array}\right) q^{k} \lambda r(r+1)+\sum_{k=0}^{\infty}\left(\begin{array}{c}
k+r-1 \\
r-1
\end{array}\right) q^{k} \beta|\gamma|-\beta|\gamma|\right. \\
& \left.+q \sum_{k=0}^{\infty}\left(\begin{array}{c}
k+r \\
r
\end{array}\right) q^{k} r(\lambda \beta|\gamma|+\lambda+1)\right] \\
& =\frac{q^{2} r(r+1) \lambda}{(1-q)^{2}}+\frac{q r(\lambda \beta|\gamma|+\lambda+1)}{1-q}+\beta|\gamma|\left[1-(1-q)^{r}\right] \text {. }
\end{aligned}
$$


Therefore the inequality 10 holds if and only if

$$
\frac{q^{2} r(r+1) \lambda}{(1-q)^{2}}+\frac{q r(\lambda \beta|\gamma|+\lambda+1)}{1-q}+\beta|\gamma|\left[1-(1-q)^{r}\right] \leq \beta|\gamma|,
$$

which is equivalent to 9 . This completes the proof.

Upon letting $\lambda=0$ and $\beta=1$, Theorem 4 yields the following result.

Corollary 5. $\mathbf{\Phi}_{q}^{r}(z)$ given by $(6)$ is in the class $\mathcal{S}(\gamma, 0,1) \subset \mathcal{S}^{*}(\gamma)$ if and only if

$$
\frac{q r}{(1-q)^{r+1}} \leq|\gamma| \text {. }
$$

Taking $\lambda=0$ and $\gamma=\beta=1$, we obtain the following corollary.

Corollary 6. $\boldsymbol{\Phi}_{q}^{r}(z)$ given by (6) is in the class $\mathcal{S}(1,0,1) \subset \mathcal{S}^{*}$ if and only if

$$
\frac{q r}{(1-q)^{r+1}} \leq 1
$$

Theorem 7. $\boldsymbol{\Phi}_{q}^{r}(z)$ given by (6) is in the class $\mathcal{R}(\gamma, \lambda, \beta)$ if and only if

$$
\frac{q^{2} r(r+1) \lambda}{(1-q)^{2}}+\frac{q r(1+2 \lambda)}{1-q}+1-(1-q)^{r} \leq \beta|\gamma| .
$$

Proof. To prove that $\boldsymbol{\Phi}_{q}^{r} \in \mathcal{R}(\gamma, \lambda, \beta)$, according to Lemma 3, it is sufficient to show that

$$
\sum_{k=2}^{\infty} k[\lambda(k-1)+1]\left(\begin{array}{c}
k+r-2 \\
r-1
\end{array}\right) q^{k-1}(1-q)^{r} \leq \beta|\gamma| .
$$

Now,using the same method as in the proof of Theorem 4, we obtain

$$
\begin{gathered}
\sum_{k=2}^{\infty} k[\lambda(k-1)+1]\left(\begin{array}{c}
k+r-2 \\
r-1
\end{array}\right) q^{k-1}(1-q)^{r} \\
=(1-q)^{r}\left[\sum_{k=2}^{\infty}\left(\begin{array}{c}
k+r-2 \\
r-1
\end{array}\right) q^{k-1} \lambda(k-1)(k-2)\right. \\
\left.\quad+\sum_{k=2}^{\infty}\left(\begin{array}{c}
k+r-2 \\
r-1
\end{array}\right) q^{k-1}(k-1)(1+2 \lambda)+\sum_{k=2}^{\infty}\left(\begin{array}{c}
k+r-2 \\
r-1
\end{array}\right) q^{k-1}\right] \\
=(1-q)^{r}\left[\begin{array}{c}
q^{2} \sum_{k=3}^{\infty}\left(\begin{array}{c}
k+r-2 \\
r+1
\end{array}\right) q^{k-3} \lambda r(r+1)+q \sum_{k=2}^{\infty}\left(\begin{array}{c}
k+r-2 \\
r
\end{array}\right) q^{k-2} r(1+2 \lambda) \\
\left.+\sum_{k=2}^{\infty}\left(\begin{array}{c}
k+r-2 \\
r-1
\end{array}\right) q^{k-1}\right]
\end{array}\right.
\end{gathered}
$$




$$
\begin{gathered}
=(1-q)^{r}\left[q^{2} \sum_{k=0}^{\infty}\left(\begin{array}{c}
k+r+1 \\
r+1
\end{array}\right) q^{k} \lambda r(r+1)+q \sum_{k=0}^{\infty}\left(\begin{array}{c}
k+r \\
r
\end{array}\right) q^{k} r(1+2 \lambda)\right. \\
\left.+\sum_{k=0}^{\infty}\left(\begin{array}{c}
k+r-1 \\
r-1
\end{array}\right) q^{k}-1\right] \\
=\frac{q^{2} r(r+1) \lambda}{(1-q)^{2}}+\frac{q r(1+2 \lambda)}{1-q}+1-(1-q)^{r} .
\end{gathered}
$$

Therefore the inequality 12 holds if and only if

$$
\frac{q^{2} r(r+1) \lambda}{(1-q)^{2}}+\frac{q r(1+2 \lambda)}{1-q}+1-(1-q)^{r} \leq \beta|\gamma| .
$$

This completes the proof.

As a special case of Theorem 7 , if we put $\lambda=0$ and $\beta=1$, we arrive at the following result.

Corollary 8. $\mathbf{\Phi}_{q}^{r}(z)$ given by $(\sqrt{6})$ is in the class $\mathcal{R}(\gamma, 0,1) \subset \mathcal{R}(\gamma)$ if and only if

$$
\frac{q r}{1-q}+1-(1-q)^{r} \leq|\gamma|
$$

Taking $\lambda=0$ and $\gamma=\beta=1$, we obtain the following corollary.

Corollary 9. $\mathbf{\Phi}_{q}^{r}(z)$ given by $(\sqrt{6})$ is in the class $\mathcal{R}(1,0,1) \subset \mathcal{R}(1)$ if and only if

$$
\frac{q r}{1-q}+1-(1-q)^{r} \leq 1
$$

\section{Integral Operators}

In this section, we will give analog results for the integral operators defined as follows:

$$
H_{q}^{r}(z)=\int_{0}^{z} \frac{\boldsymbol{\Phi}_{q}^{r}(t)}{t} d t
$$

where $\boldsymbol{\Phi}_{q}^{r}(t)$ is given by 6 .

Theorem 10. $H_{q}^{r}(z)$ given by 13) is in the class $\mathcal{S}(\gamma, \lambda, \beta)$ if and only if

$\frac{\lambda q r}{(1-q)}+\frac{(1-\lambda)(\beta|\gamma|-1)(1-q)}{q(r-1)}\left[1-(1-q)^{r-1}\right]-\beta|\gamma|(1-q)^{r}+\lambda \beta|\gamma|+1-\lambda \leq \beta|\gamma|$. 
Proof. From [13, we can write

$$
H_{q}^{r}(z)=\int_{0}^{z} \frac{\boldsymbol{\Phi}_{q}^{r}(t)}{t} d t=z-\sum_{k=2}^{\infty}\left(\begin{array}{c}
k+r-2 \\
r-1
\end{array}\right) q^{k-1}(1-q)^{r} \frac{z^{k}}{k} .
$$

According to Lemma 2, it is enough to show that

$$
\sum_{k=2}^{\infty} \frac{[\lambda(k-1)+1](k+\beta|\gamma|-1)}{k}\left(\begin{array}{c}
k+r-2 \\
r-1
\end{array}\right) q^{k-1}(1-q)^{r} \leq \beta|\gamma| .
$$

Using the assumption (14), a simple computation shows that

$$
\begin{aligned}
& \sum_{k=2}^{\infty} \frac{[\lambda(k-1)+1](k+\beta|\gamma|-1)}{k}\left(\begin{array}{c}
k+r-2 \\
r-1
\end{array}\right) q^{k-1}(1-q)^{r} \\
& =(1-q)^{r}\left[\sum_{k=2}^{\infty}\left(\begin{array}{c}
k+r-2 \\
r-1
\end{array}\right) q^{k-1} \lambda(k-1)+\sum_{k=2}^{\infty}\left(\begin{array}{c}
k+r-2 \\
r-1
\end{array}\right) q^{k-1}(\lambda \beta|\gamma|-\lambda+1)\right. \\
& \left.+\sum_{k=2}^{\infty}\left(\begin{array}{c}
k+r-2 \\
r-1
\end{array}\right) q^{k-1} \frac{(1-\lambda)(\beta|\gamma|-1)}{k}\right] \\
& =(1-q)^{r}\left[q \sum_{k=2}^{\infty}\left(\begin{array}{c}
k+r-2 \\
r
\end{array}\right) q^{k-2} \lambda r+\sum_{k=2}^{\infty}\left(\begin{array}{c}
k+r-2 \\
r-1
\end{array}\right) q^{k-1}(\lambda \beta|\gamma|-\lambda+1)\right. \\
& \left.+\frac{(1-\lambda)(\beta|\gamma|-1)}{q(r-1)} \sum_{k=2}^{\infty}\left(\begin{array}{c}
k+r-2 \\
r-2
\end{array}\right) q^{k}\right] \\
& =(1-q)^{r}\left\{\lambda q r \sum_{k=0}^{\infty}\left(\begin{array}{c}
k+r \\
r
\end{array}\right) q^{k}+(\lambda \beta|\gamma|-\lambda+1)\left[\sum_{k=0}^{\infty}\left(\begin{array}{c}
k+r-1 \\
r-1
\end{array}\right) q^{k}-1\right]\right. \\
& \left.+\frac{(1-\lambda)(\beta|\gamma|-1)}{q(r-1)}\left[\sum_{k=0}^{\infty}\left(\begin{array}{c}
k+r-2 \\
r-2
\end{array}\right) q^{k}-1-q(r-1)\right]\right\} \\
& =\frac{\lambda q r}{(1-q)}+(\lambda \beta|\gamma|-\lambda+1)\left[1-(1-q)^{r}\right] \\
& +\frac{(1-\lambda)(\beta|\gamma|-1)}{q(r-1)}\left[(1-q)-(1-q)^{r}-q(r-1)(1-q)^{r}\right] \\
& =\frac{\lambda q r}{(1-q)}+\frac{(1-\lambda)(\beta|\gamma|-1)(1-q)}{q(r-1)}\left[1-(1-q)^{r-1}\right]-\beta|\gamma|(1-q)^{r}+\lambda \beta|\gamma|+1-\lambda .
\end{aligned}
$$

From (14), we conclude that $H_{q}^{r}(z) \in \mathcal{S}(\gamma, \lambda, \beta)$. This completes the proof.

Theorem 11. $H_{q}^{r}(z)$ given by 13$)$ is in the class $\mathcal{R}(\gamma, \lambda, \beta)$ if and only if

$$
\frac{q r \lambda}{(1-q)}+1-(1-q)^{r} \leq \beta|\gamma| \text {. }
$$


Proof. Since

$$
H_{q}^{r}(z)=z-\sum_{k=2}^{\infty}\left(\begin{array}{c}
k+r-2 \\
r-1
\end{array}\right) q^{k-1}(1-q)^{r} \frac{z^{k}}{k}
$$

according to Lemma 3 , it is enough to show that

$$
\sum_{k=2}^{\infty} \frac{k[\lambda(k-1)+1]}{k}\left(\begin{array}{c}
k+r-2 \\
r-1
\end{array}\right) q^{k-1}(1-q)^{r} \leq \beta|\gamma| .
$$

Using the assumption (17), some simple computations shows that

$$
\begin{aligned}
\sum_{k=2}^{\infty} \frac{k[\lambda(k-1)+1]}{k}\left(\begin{array}{c}
k+r-2 \\
r-1
\end{array}\right) q^{k-1}(1-q)^{r} & \\
= & (1-q)^{r}\left[\sum_{k=2}^{\infty}\left(\begin{array}{c}
k+r-2 \\
r-1
\end{array}\right) q^{k-1} \lambda(k-1)+\sum_{k=2}^{\infty}\left(\begin{array}{c}
k+r-2 \\
r-1
\end{array}\right) q^{k-1}\right] \\
= & (1-q)^{r}\left[q \sum_{k=2}^{\infty}\left(\begin{array}{c}
k+r-2 \\
r
\end{array}\right) q^{k-2} \lambda r+\sum_{k=2}^{\infty}\left(\begin{array}{c}
k+r-2 \\
r-1
\end{array}\right) q^{k-1}\right] \\
=(1-q)^{r} & \left.q \lambda r \sum_{k=0}^{\infty}\left(\begin{array}{c}
k+r \\
r
\end{array}\right) q^{k}+\sum_{k=0}^{\infty}\left(\begin{array}{c}
k+r-1 \\
r-1
\end{array}\right) q^{k}-1\right] \\
= & \frac{q r \lambda}{(1-q)}+1-(1-q)^{r}
\end{aligned}
$$

From (17), we conclude that $H_{q}^{r}(z) \in \mathcal{R}(\gamma, \lambda, \beta)$. This completes the proof.

Author Contribution Statements All authors contributed equally to the planning, execution, and analysis of this research paper.

Declaration of Competing Interests No potential conflict of interest and there is no funding was reported by the authors.

\section{REFERENCES}

[1] Altınkaya, Ş., Yalçın, S., Poisson distribution series for analytic univalent functions, Complex Anal. Oper. Theory,12 (2018), 1315-1319. https://doi.org/10.1007/s11785-018-0764-y

[2] Altintaş, O., Özkan, Ö., Srivastava, H. M., Neighorhoods of a class of analytic functions with negative coefficient, Applied Mathematics Letters, 13(3) (2000), 63-67. https://doi.org/10.1016/S0893-9659(99)00187-1

[3] Çakmak, S., Yalçın, S., Altınkaya, Ş., Some connections between various classes of analytic functions associated with the power series distribution, Sakarya Üniversitesi Fen Bilimleri Enstitüsü Dergisi, 23(5) (2019), 982-985. https://doi.org/10.16984/saufenbilder.552957

[4] El-Deeb, S.M., Bulboaca, T., Dziok, J., Pascal distribution series connected with certain subclasses of univalent functions, Kyungpook Mathematical Journal, 59(2) (2019), 301-314. https://doi.org/10.5666/KMJ.2019.59.2.301

[5] Duren, P. L., Univalent Functions, Grundlehren der Mathematischen Wissenschaften 259, Springer-Verlag, New York, 1983. 
[6] Halim, S. A., On a class of functions of complex order, Tamkang Journal of Mathematics, 30(2) (1999), 147-153. https://doi.org/10.5556/j.tkjm.30.1999.4221

[7] Murugusundaramoorthy, G., Vijaya, K., Porwal, S., Some inclusion results of certain subclass of analytic functions associated with Poisson distribution series, Hacettepe Journal of Mathematics and Statistics, 45(4) (2016), 1101-1107. https://doi.org/10.15672/HJMS.20164513110

[8] Murugusundaramoorthy, G., Subclasses of starlike and convex functions involving Poisson distribution series, Afr. Mat., 28 (2017), 1357-1366. https://doi.org/10.1007/s13370-017-0520-x

[9] Nazeer, W., Mehmood, Q., Kang, S. M., Haq, A. U., An application of Binomial distribution series on certain analytic functions, Journal of Computational Analysis and Applications, 11 (2019).

[10] Nasr, M. A., Aouf, M. K., Starlike functions of complex order, Journal of Natural Sciences and Mathematics, 25 (1985), 1-12.

[11] Owa, S., Notes on starlike, convex, and close-to-convex functions of complex order, in : H. M. Srivastava and S. Owa (Eds.), Univalent Functions, Fractional Calculus, and Their Applications, 199-218, Halsted Press. (Ellis Horwood Limited, Chichester), John Wiley and Sons, New York, Chichester, Brisbane and Toronto, 1989.

[12] Porwal, S., An application of a Poisson distribution series on certain analytic functions, Journal of Complex Analysis, 1-3 (2014), Article ID 984135. http://dx.doi.org/10.1155/2014/984135

[13] Porwal, S., Kumar, M., A unified study on starlike and convex functions associated with Poisson distribution series, Afr. Mat., 27 (2016), 1021-1027 https://doi.org/10.1007/s13370016-0398-z.

[14] Silverman, H., Univalent functions with negative coefficients, Proc. Am.Math. Soc., 51 (1975), 109-116. https://doi.org/10.1090/S0002-9939-1975-0369678-0

[15] Wiatrowski, P., The coefficients of a certain family of holomorphic functions, Zeszyty Nauk. Uniw. Lodz Nauk. Mat.-Przyrod, (Ser.II), 39 (1971) 75-85. 